blood, and there was a mark of blood on the wall where the head was resting. Between the front door and the body and about one or two feet from it were two small pools of clotted blood. Neither the hands nor the shnes of the deceased were bloody, and there were no bloody footmarks, nor any blood on any other part of deceased, nor on her clothes. There were no other marks of violence visible, nor any signs of a struggle whatever. Beneath the body was found an old pewter tall candlestick; this had no recent crack or injury, nor blood-stain. Near the pools of blood was a candle, which had been lighted at some time; it was crushed by pressure, and adhering to it was a coil of twine. I' was adberent to the floor and was discoloured, perhaps by blood. No weapon and nothing else unusual was found on the ground floor. Upstairs it was found that the bedroom was undisturbed, the bed had not been slept in. On enter. ing the front downstairs room the husband was found lying on a sofa apparently insensible from drink. Bottles and glasses containing small quantities of beer and rum were on the table. The windows were not curtained nor were the shutters closed, nor was the gas alight. Everything appeared as if the room had not been lighted up the previous evening. There were no signs of any struggle having occurred, and no marks of blood on anything, except that on the mantelpiece was a clay pipe which bore stains of bloody fingers. In the back kitchen sink were two small vessels containing fluid : in one (a small tureen) the liquid was pink, and was found to be stained with blood. The husbaud, who was dressed in every.day clothes, was aroused and told of his wife's death. He only answered, "I know nothing about it." Blood-stains were found on his hands and on his face, which bore marks of scratches and some shaving scars ; here were blood-stains also on his waistcoat and on the front of his trousers, on one stocking, and on one shirtsleeve, but the coat was clean, and the inside of the coatsleeve corresponding to the stained shirt-sleeve was clean. His clothing was not torn, and there were no marks of any struggte having taken place. The inspector of police $n \in X$ arrived, and to him the husband stated, after being cautioned, that " he had gone to pick her up; she had asked him for some brandy; he had gone down to fetch it, but had fallen asleep and forgotten all about it." The husband, however, was still partially insensible from drink throughout the moraing. He was arrested and charged with causing the death, aud remanded until the verdict of the coroner's jury had been given. The effect of which proceeding was of course to prevent his giving any evidence at the inquest. On an examination of his clothing it was found that the blood-stain of the trousers- bore evidence of an attempt having been made to wash out the stains, but this was not the case with the other stains on the clothes.

At a post-mortem examination of the body, made by Mr. Lloyd, twenty-six hours after the discovery of the body, the following notes were taken :-Age about sixty, body well nourished, weight about twelve stone; no external mariss of violence except the wound of the scalp before mentioned, which was of rectangular shape, its two sides about one-third of an inch long each; it was situated just above the oceipital protuberance, it did not extend down to the bone. There was some bruising of the scalp around it, but no fracture of the skull, and the brain was found to be quite healthy and free from injury. The lungs were congested and emphysematous; there were some old adhesions on the right side. The diaphragm was ruptured, and the greater part of the stomach and the spleen had passed through the rent into the left pleural cavity. The ribs, from the third to the ninth on the left side, were all broken, the fractures forming an even line extending down wards and outwards from about one inch external to the junction of the third rib, with its costal cartilage. The broken ends of the ribs had not punctured the pleura, but there was extravasation of blood around them. The heart exhibited concentric hypertrophy of the left ventricle; there were atheromatous patches on the intima. The lining membrane of the stomach was reddened, the liver cirrhosed, the kidneys more vascular than usual, their capsules adherent, and their surface nodular.

In answer to questions put by the coroner the medical witness stated that the effects of such terrible injuries to the diaphragm and displacement of the viscera must have been to cause sudden death, possibly without any andible cry; that the injury must have been caused by a sudden violent compression of the body against a resisting even edge, such as the edge of a stair or a banister rail, or else by an excessively violent blow by a long blunt weapon. Further, that the scalp wound was not sufficient to cause the death although it might account for all the blood found. He did not consider the scalp wound had been caused by the candlestick produced, because it was uninjured and clean, and because it was of too brittle a metal not to show signs of violence. And, finally, considering the formation of the staircases precluding the idea of a fall from a height, and in the absence of any weapon, he was unable to assign any probable cause of the injuries. Being questioned as to the time at which death occurred, he stated that it was almost im. possible to guess, from the fact that the body was in a sense absolutely cold and the cadaveric rigidity perfect; the deceased must have been dead for some hours before 9 A.M. on Monday. She might have been dead since sunset on Sunday evening. He considered, however, that it was certain that the body had been moved since the rigor mortis had set in, because when found the stiffened neck was supporting the head; this appearance could not have existed if the person had died in the situation where she was found. Hence he concluded that some person, probably the blood-stained husband, had moved the body some hours after death from the position in which she had died and stiffened to where the body was found. 'The coroner's jury having been assured that no further evidence was likely to be fortheoming unavoidably returned a verdict of "Death from injuries described by the medical witness, but how these had been caused there was no evidence to show." The magistrate having heard the verdict of the coroner's jury, dismissed the husband from custody immediately, and so rests at present this mysterious case.

We cannot but think that if such cases as this one were more often reported in the medical journals much light might be thrown on the undecided questions of how soon after death the rigor mortis comes on, and how long it may last, taking into consideration the mode of deatb, the bodily bealth of the deceased, the presence or absence of intoxica. tion, and a vast number of other important medico.legal conditions, and we hope that the publication of this case may lead medical coroners and witnesses to accumulate a body of evidence which will enable medical jurists to cast a clear light on many such disputed topics.

\section{ON PTOMAINES, OR CADAVERIC ALKALOIDS.}

\section{By R. N. WOLFENDEN, B.A., M.B.CANTAB., F.C.S., LECTURER ON PHYSTOLOGY IN THE CHARING-CROSS HOSPITAL} MEDICAL SCHOOL.

Arong the many decompositions of proteids none are of more interest than that series of changes that give rise to the production of ptomaines, or cadaveric alkaloids. Whether regarded from a forensic point of view, or as pathological plenomena, they are of surpassing interest. A recent trial for murder brought the name of "ptomaine" into prominence, and the counsel who made use of the facts already known only to specialists for their defence undoubtedly exhilited much cleverness. Considering the importance of the subject in pathological chemistry, and the little that is known about it, except to those of us who have worked more or less in this direction, I shall endeavour to give a short résumé of the facts already pus before the scientific public.

Though the term " ptomaine" was first applied by Selmi to these bodies to indicate that they were of cadaveric origin, their distribution is no longer to be limited to the cadaver, but investigations are rendering it more and more probable that alkaloids of a poisonous nature occur in certain pathological conditions, and possibly also normally, as a product of change of living tissue. Selmi's first observations were made on corpses dead of arsenical poisoning, and it is of interest to refer to his methods. The liquid under observation was made alkaline with baryta water, and then extracted with ether; acicular crystals were produced, which precipitated the principal reagents that alkaloids answer to, with the exception of platinum chloride. Selmi's second attempt was made by extracting the liquid with aqueons alcohol, then making alkaline with baryta, shaking with ether, ridding of the ether by spontaneous evaporation and distillation, treating the turbid liquid thus left by wateracidulated with acetic acid, filtering and evaporating to dryness; then taking up the residue with water, again rendering alka. 
line with baryta and extracting with ether, and repeating the process till the liquid became colourless. The alkaloid thus extracted was so strongly poisonous as to destroy frogs with the greatest rapidity. Both of these extracts were free from arsenic. Suhsequently Selmi extracted from the stomach of a hog which had been kept on arsenious acid a compound of an alkaloid (ptomaine) and arsenic, of an intensely poisonous nature, and resembling very much the vegetal alkaloid strychnia. As to their manner of production, Selmi supposed them to be products of putrefaction of organised nitrogenous material. They are variable in their nature according to the length of time after death, and though not present within a few hours after death in any appreciable quantity, they slowly develop with advancing decomposition. Indeed, it is only with extreme difficnlty that in medien-legal cases they can be distinguished from true vegetal alkaloids, if the post-mortem examination be delayed, but probably, if made within twenty-four to fortyeight hours after death, any alkaloid found then will be a vegetal and not a cadaveric one. In general these ptomaines are oftenest amorphous in form and alkaline in reaction. They are for the most part volatile and easily alterable. They form crystalline salts with acids as a rule. The addition of acids to them usually changes them, with the production of pleasant adours, like orange-flower, musk, \&c. Allowed to oxidise by contact with atmospheric air, they emit disagreeable cadaveric or urinous odours.

Some of these ptomaines are not poisonous, but most possess strongly toxic characters, and cause profound symptoms when injected under the skin-such as paralysis, more or less complete, of hinder extremities, dilatation of pupils, convulsions, muscular flaccidity, slowing or accele ration of the heart, loss of cutaneous sensibility and of muscular contractility, possessing many of the characteristic physiological actions of muscarin or atropin. They answer to nearly the same reactions as the vegetal alkaloids, some being precipitated by platinic chloride, gold chloride, mercuric chloride or taunic acid, \&c.; and all have a strongly reducing action on potash ferridcyanide. They yield colour reactions with sulphuric acid (red), iodic acid and sulphuric acid (violet), nitric acid (yellow), \&c. Their physiological properties are really their only characteristic properties, distinguishing them from other alkaloids. These ptomaines are met with under the following conditions :-

1. As a constituent of normal tissues or juices.-In this case they are products of tissue metamorphosis. (a) If from twenty to thirty cubic centimetres of fresh saliva be taken and evaporated over a water bath to dryness, and the residue extracted with boiling water and finally filtered, a toxic substance is obtained, which is strongly reducing, and converts ferridcyanide of potash, along with one or two drops of ferrichloride, into "Prussian blue." Injected into the thigh of a frog with a Pravaz syringe, it kills the animal in a longer or shorter time. (b) Snake poison differs from human saliva only in the intensity of its action. Gautier has extracted from the poison of trigonocephalus and naja, two distinct alkaloids, and a third which resembles either an alkaloid or an albuminous compound. (c) From normal urine Gantier asserts to have obtained a fixed oxidisable alkaloid, which forms a crystalline chloride, and also a crystalline double salt with platinic and gold chloride. This alkaloid is very poisonous, killing quickly with systolic standstill of the heart, stupor, and paralysia. Though Gautier's facts are unquestionable, there is a fallacy underlying his explanation of them that renders it valueless.

2. In certain pathological conditions, recent observations of Selmi's on what he calls pathological basis would seem to show :- $(\alpha)$ In the urines of patients suffering from progressive paralysis there are two volatile bases: the one like nicotin, the other like coniin. (b) In the urine voided during a case of interstitial pneumonia were two alkaloidal bases: one having the odour of stinking fish, the other of ammonia. (c) $T$ wo similar bases were found in the urine of patients with abdominal typhus. (d) In tetanus a base-like coniin was discovered in the urine. (e) In the urine of "miliary fever" an alkaloidal base having the odour of stinking fish has been observed.

3. As acadaveric or artificial production. - There can be no further doubt that these bodies are largely produced in the process of decomposition af nitrogenous or proteid tissnes. Since Selmi first described their production his facts have been abundantly confirmed and extended. There are many varieties of them, and they vary much in their nature, according as the length of time after death is long or short. They are, however, of slow production naturally, point referred to before, and to be borne in mind in conducting medico-legal inquiries. Stinking fish, bad meat, \&.., all contain poisonous principles which can be extracted after the manner of ptomaines. The gastro-inte-tinal irritation and profound toxic symptoms produced by the ingestion of bad food are probably at the bottom processes of alkaloidal poisoning. On this subject I shall have more to say on another occasion. It is even possible that many so-called uræmic phenomena are produced by the retention within the body of these alkaloidal poisons; and, further, that the products of gastric digestion contain poisonous properties which, imperfectly excreted, may become toxic. There is a statement by Balduino Bocci that normal urine, especially after meal times, if injected into frogs destroys them.rapidly. The observations of Gautier that an alkaloid of highly poisonous nature is to be obtained from normal haman urine is of importance with reference to this po $0^{\circ} \mathrm{x}$.

Brieger has obtained by the decomposition of neurin final products of trimetbylamine, and a volatile body giving the iodoform reaction, which he suggests may be identical with those volatile substances in urine which give the iodoform reaction. By decomposition of nearin and albumen toxic bodies may be obtained much resembling muscarin. Both from freshly prepared peptone made by the action of gastric juice on pure fibrin, and from stinking peptone by boiling with caustic soda, evaporating, extracting, and purifying, toxic alkaloids can be obtained which kill frogs and rabbits. in a few minutes, Putrefying casein, brain substance, liver, and muscle also yield these products. In the laboratory I have confirmed the observations of others as to the production of these substances, and I have also confirmed the statement of Gautier as to the production of a toxic principle from ordinary fresh saliva, which are very destructive to $\$$ mall animals. The extension of these observations to the investigation of snake poison and the salivary poisons of certain animals in the pathological state (rabies, \&c.) becomes of extreme import. The investigations of Weir-Mitchell on the production of snake-poison are of extreme interest in connexion with this point, indicating as they do the discovery of three toxic principles in serpents' venom : the one resembling a peptone, the other a globulin, and the third serum albumen. ${ }^{1}$

Some of these ptomaines are poisonous, some non-poisonous; and it is common to find a poisonous and a non-poisonous moiety linked together. Some are volatile and crystalline, others non-volatile and non-crystalline, some precipitated by ether from acid or alkaline solutions, and some only precipitated by amyl, alcohol, or chloroform. With regard to tests, they answer to many of those usually employed for veoetal alkaloids, giving a dark colour with tannin, a thick yellow precipitate with potash cadmium iodide or potasso. mercuric iodide, with cadmium or bismuth iodide a red pre cipitate, with gold a mercuric chloride, a precipitate, and some of them precipitating platinic chloride. The vast majority are strongly reducing. This fact was put forward by Brouardel and Boutmy as a distinctive test for the presence of ptomaines. The base extracted from the body is converted into a sulphate, and to a little put into a watch glass which contains ferridcyanide of potash a few drops of ferric chloride are added. If a ptomaine be present there is a reduction of the ferridcyanide and the formation of Prussian blue. Brouardel and Boutmy asserted this reaction to be given only by ptomaines, morphia, and perhaps veratria, and to be in a manner characteristic. It has since been proved by Tauret, Gautier, and others that many*vegetal alkaloids yield this test (aconite, digitaline, ergotinine, byoscyamine, \&c.), and also many artificial alkaloids (anitine, methylaniline, phenylamine, collidine, \&c.). Even gelatine soluiions and solutions of extract of meat give this Prussian blue reaction, so that it cannot for an instant be taken as characteristic of the ptomaines. No chemical test has as yet been devised that is at all crucial, and the general reactions, taken along with their physiological peculiarities alone, can be taken as evidence of the presence of a ptomaine.

The ptomaines so far described are:-1. Ptomaines, like atropin and hyoscyamine (Sonnenschein and Quelzer), crystalline, and which dilate the pupil and accelerate the heart. They are obtained from putrescent albuminous fluids. 2. An alkaloid from decomposing yeast (Schmiedeberg and Bergmann), which the authors call sepsin, and which resembles strychnia. 3. An alkaloid resembliog morphia in its tests, but not in its physiological properties (Selmi). 4. One 
agreeing with delphinin, and which by warming with phosphoric acid gives a red colour. It does not agree physiologically. 5. One resembling strychnia in its behaviour towards sulphuric acid and potash bichromate, but not causing tetanus (Ciotto). 6. Alkaloids like muscarin (Gautier, Brieger, \&c.). 7. Alkaloids like coniin, colourless, leaving a sharp taste like tobacco, and consisting of a poisonous and non-poisonous moiety, one part volatile the other non-volatile; one soluble and precipitated by warmth, like coniin, the other not. 8. Alkaloids resembling parvolin and hydrocollidin (Gautier and Stard), and one like collidin (Neucki).

As to the manner of production of these bodies chemically, I do not propose here to enter. It could only at present be speculative. I would sum up briefly as follows :-

1. There are developed in the body, post mortem, poisons of an alkaloidal character, and which can be obtained also by decomposition of albumen, peptone, casein, muscle, brain, \&c. Moreover, they seem to be present in some normal secretions (saliva and urine).

2. These cadaveric alkaloids may be mistaken, post mortem, for vegetal poisons administered with evil intent, but if the body be examined within from twenty-four to forty-eight hours after death, any alkaloid there found would be strong presumptive evidence of poison, and not ptomaine. After a couple of days it may be a matter of doubt.

3. There is no satisfactory test surely indicating the presence of a ptomaine. Physiological characters must be taken in conjunction with chemical tests.

4. Probably the production of ptomaines within the living body may be the pathological cause of many obscure conditions, especially those following on poisoning by bad food, such as stale fish, \&c.

Welbeck-street.

\section{ON THE}

\section{CAUSES OF PUERPERAL ECLAMPSTA.}

BY HENRY CORBY, M.A., M.D., PHYSICIAN TO THE CORK MATERNITY.

ON the 3lst of March last I saw Mrs. B_. She was suffering from puerperal eclampsia. She had had a convulsion earlier in the morning, and two or three afterwards. Draughts containing hydrate of chloral and bromide of potassium were prescribed, as well as purgatives by the mouth and rectum. The lower extremities were cedematous, and the urine contained so much albumen, that on the application of heat and nitric acid it became almost a solid mass. The following day the albumen was diminished by about one-half, but on the second day hæmaturia occurred. On inquiring into the case, I learned that the patient was six months pregnant, that a week previous to the convulsions she experienced a dimness of sight, and that pressure in the abdomen caused pain. During an interval between the convulsions, on making an external examination, I noticed that the uterine tumour was not nearly as large as might be expected at six montbs, and, more remarkable still, I found that the fundus had not the usual semilunar outline, but appeared to be bound down in the centre as if a band had been passed from above downwards towards the cervix, and prevented the uterus from expanding upwards. On reflection it occurred to me that the condition of the uterus may have had a good deal to do with the production of the convulsions, and since then, having heard of another case of eclampsia in the neighbourhood, I have questioned the medical gentleman in charge, and learned from him that in his patient there was a previous history of oöphoritis, probably causing adhesions sufficient to more or less bind down the uterus, and the abdominal enlargement was not as great as might have been expected in a woman at her period of pregnancy-within a fortuight of her full time.

It may of course be considered rash to theorise as to the cause of a disease from the facts observed in one or two instances, but as I fancy my idea of the causation enables me to explain the various phenomena of this disease, I am induced to put my views before the profession. Dr. MacDonald states that by actual post-mortem examination he Las demonstrated that there is anæmia of the cerebro-spinal centres in patients suffering from puerperal eclampsia. This may be, and probably is, one of the direct causes of the convulsions, but the question remains, What produces the anæmia? - in other words, What are the more remote causes of the disease? Braun states that urea and other excrementitious products retained in the blood in connexion with the presence of albumen in the urine are the factors in the production of the convulsions, and that the albuminuria is the result of Bright's disease. Frerichs considers that car bonate of ammonia produced by the decomposition of urea is the poisonous agent. But with regard to these theories it has been pointed out that puerperal convulsions occur when there has been no albumen in the urine previously, and that the albuminuria may be the result rather than the cause of the convalsions. It has been further urged that many pregnant women have albumen in the urine temporarily and that if the presence of the albumen were such an all powerful causative element, we should have convulsions occurring more frequently.

Now, the view 1 take of the matter is that the uterus, owing to adhesions or through some other defect, cannot expand upwards, and then it presses unduly in every other direction ; amongst others backwards. We have thus pres. sure exerted on the large vessels behind the uterus, and the blood is delayed in its passage through the kidneys. Albuminuria results just as it does in ordinary cases of dilated heart. All this time the foetus is growing, becomes unduly large for the uterus, and acts as a foreign and irritating body. Convulsions result from the irritation just as they do when there are worms in the intestine, and just as they do in a case of traumatic tetanus. We know, too, that many pregnant women are in a highly sensitive condition and that nervous influences act and react much more readily than in the unimpregnated female. I believe, then, that for the production of puerperal eclampsia there are two causes operating, either separately or together-namely retained excrementitious urinary products and the irritation of the proportionately large fcetus in the uterus. If this view be correct, we can explain several of the phenomena in connexion with the disease. For instance, the mere presence of albumen in the urine is not necessarily sufficient to cause convulsions in every woman, as the correspondeng retention of excrementitious matter may not be sufficiently great, or might require the addition of the uterine irritation to produce the convulsions. Again, the delay in the occurrence of the convulsions, in some instances till after delivery, may be accounted for by the consideration that the excrementitious poisons had been accumulating, and the uterine irritation was gradually affecting the nervous centres till they became charged and the explosion took place. We can easily understand, too, why it is that, as a rule, the convulsions diminish in frequency, or cease altogether, after delivery ; for then the backward pressure on the vessels and the uterine irritation are both removed. It is easy also to explain how it is that in so many instances labour occurs prematurely, and why it is that the foetus is so frequently born dead. As we know that, generally speaking, the introduction of the hand into the uterus excites con. tractions, so the impinging of the foetus against the imperfectly expanded uterus will develop strong expulsive action, and in addition the convulsive contractions to which the uterus, in common with other parts of the body, is subjected, must have a tendency to bring on labour. With reference to the death of the foetus, it is apparent from what has been said above that many things contribute to the pro. duction of that event-namely, the poisoned condition of the blood ; the almost direct pressure of the uterus, owing to the proportionately small quantity of the protecting amniotic fluid; the tonic spasms followed so closely by the clonic spasms, and one or both sometimes so prolonged that the interval of quiescence, such as occurs between the ordinary labour pains, does not supervene till the life of the child is sacrificed.

If these views of the direase be correct, the provious treatment of a patient suffering from puerperal eclampsia would require a good deal of consideration and attention. Cork.

The Late Dr. Loftie Stoney, of Dublin.At a largely attended meeting of students connected with the Carmichael College of Medicine, the institution where Dr. Stoney held the appointment of lecturer on anatomy, it was decided to erect a suitable memorial to the memory of the deceased gentleman. 